\title{
Speckle tracking echocardiography: another step towards early detection of septic myocardial dysfunction?
}

\author{
Elio Antonucci ${ }^{*}$ id and Sara Agosta \\ See related research by Ng et al., http://ccforum.biomedcentral.com/articles/10.1186/s13054-016-1327-0
}

We read with interest the recent paper by $\mathrm{Ng}$ et al. [1] dealing with the use of speckle tracking echocardiography (STE) in septic myocardial dysfunction. In this study, the authors found that STE can detect a left ventricular impairment more sensitively than conventional echocardiography in septic shock patients. The study offers new perspectives to improve diagnosis of septic myocardial dysfunction, and the authors should be commended for their work. However, some important hemodynamic issues should be further discussed to better interpret the results.

Firstly, the cardiac preload was represented by the right atrial pressure (RAP), estimated by the inferior vena cava (IVC) diameter and the presence of inspiratory collapse. Results proved that the RAP was higher in the study group of patients than in the control group $(7.4 \mathrm{mmHg}$ vs $5.9 \mathrm{mmHg}, p=0.017$ ), even if the amount of fluids given in the first hours of shock were not known. Moreover, if we consider that only $16 / 33$ patients (48\%) were mechanically ventilated, use of the IVC diameter could be debatable to predict the cardiac preload in spontaneous breathing patients, as reported previously [2].

Secondly, no information about the dosage and type of vasopressor/inotropic support was provided. In patients supported by catecholamines, STE might not be accurate because of altered strain rates induced by high doses of dobutamine [3].

In addition, mean cardiac output (CO) and the afterload measures were unexpectedly similar both in the study group and in the control group of patients $(\mathrm{CO}=5.88 \mathrm{~L} / \mathrm{min}$ vs $5.48 \mathrm{~L} / \mathrm{min}, p=0.40$; SVR $=1090$ dynes $\bullet \mathrm{sec} / \mathrm{cm}^{5}$ vs 1194 dynes $\bullet$ sec $/ \mathrm{cm}^{5}, p=0.303$ ) despite the presence of distributive shock in the first group. Moreover, relevant data about tissue perfusion and microcirculatory dysfunction, such as lactate levels and venous-to-arterial carbon dioxide differences, were not reported.

Lastly, the authors evaluated both mechanically ventilated patients and nonventilated patients in the study group. We know that the effects of mechanical ventilation on cardiac function and hemodynamics are complex and are characterized by reduced venous return (due to an increase in RAP), increased pulmonary vascular resistance (PVR), and reduced CO [4]. However, the present study is lacking in some important information about the positive end-expiratory pressure (PEEP), tidal volume, and respiratory rate, data that, instead, were accurately reported in the previous echocardiography works in this field [5].

In conclusion, further prospective studies are warranted to investigate the clinical utility of STE in septic myocardial dysfunction.

\section{Authors' response}

Pauline Yeung Ng, Wai Ching Sin and Andrew Kei-yan Ng

We thank Dr Antonucci and Dr Agosta for their interest in our research article [1] and their comments.

We reported RAP estimated by the IVC diameter and collapsibility as a measure of cardiac preload. Although

\footnotetext{
* Correspondence: elio.antonucci@yahoo.it

Intermediate Care Unit, Emergency Department, Guglielmo da Saliceto Hospital, Piacenza, Italy
}

a difference in RAP between the two groups existed (7.4 $\mathrm{mmHg}$ vs $5.9 \mathrm{mmHg}, p=0.017$ ), the absolute difference of $1.5 \mathrm{mmHg}$ is probably clinically negligible. Left ventricular end diastolic volume (LVEDV) is a better of LV preload [6], and was similar in the two groups (74.1 $\mathrm{ml}$ vs $74.7 \mathrm{ml}, p=0.90)$, suggesting similar 
preload conditions. The effect of preload on strain parameters is not completely understood, but small-scale studies have shown cardiac strain to be relatively load independent [7].

Regarding the use of vasopressor or inotropic support, all patients received noradrenaline as the initial drug of choice during resuscitation. No patients received dobutamine during the course of treatment.

We attribute the similar $\mathrm{CO}$ and systemic vascular resistance (SVR) in the study and control groups to two factors. Firstly, instead of healthy individuals, the control group was a group of patients with sepsis. These patients would have similarly raised CO and reduced SVR, albeit to different degrees. Secondly, because of the time-consuming nature of performing strain analysis, all echocardiographic assessments were done after initial stabilization, which included vasopressor administration in the context of the distributive shock. Although logistically difficult, it will be an excellent research topic to assess the speckle tracing during the initial period of septic shock.

Finally, the effect of mechanical ventilation on hemodynamics was not explored in depth in our study. Subgroup analysis was not performed due to the relatively small number of patients $(16 / 33)$ on mechanical ventilation. However, a similar right ventricular index of myocardial performance between the two groups speaks against a significant effect of mechanical ventilation on right ventricular function.

\section{Abbreviations}

$\mathrm{CO}$, cardiac output; IVC, inferior vena cava; LVEDV, left ventricular end diastolic volume; PEEP, positive end-expiratory pressure; PVR, pulmonary vascular resistance; RAP, right atrial pressure; STE, speckle tracking echocardiography; SVR, systemic vascular resistance

Published online: 18 August 2016

\section{References}

1. Ng PY, Sin WC, Ng AK, Chan WM. Speckle tracking echocardiography in patients with septic shock: a case control study (SPECKSS). Crit Care. 2016:20:145.

2. Muller L, Bobbia X, Toumi M, Louart G, Molinari N, Ragonnet B, et al. Respiratory variations of inferior vena cava diameter to predict fluid responsiveness in spontaneously breathing patients with acute circulatory failure: need for a cautious use. Crit Care. 2012;16:R188.

3. Abraham TP, Pinheiro AC. Speckle-derived strain a better tool for quantification of stress echocardiography? J Am Coll Cardiol. 2008;51:158-60.

4. Fougères $\mathrm{E}$, Teboul $\mathrm{J}$, Richard C, Osman D, Chemla D, Monnet X. Hemodynamic impact of a positive end-expiratory pressure setting in acute respiratory distress syndrome: importance of the volume status. Crit Care Med. 2010;38:802-7.

5. Vieillard Baron A, Schmitt JM, Beauchet A, Augarde R, Prin S, Page B, et al. Early preload adaptation in septic shock? A transesophageal echocardiographic study. Anesthesiology. 2001;94:400-6.

6. Sasai T, Tokioka H, Fukushima T, Mikane T, Oku S, Iwasaki E, Ishii M, Mieda H, Ishikawa T, Minami E. Reliability of central venous pressure to assess left ventricular preload for fluid resuscitation in patients with septic shock. J Intensive Care. 2014;2(1):58.

7. Weber U, Base E, Ristl R, Mora B. Effect of preload alterations on left ventricular systolic parameters including speckle-tracking echocardiography radial strain during general anesthesia. J Cardiothorac Vasc Anesth. 2015;29(4):852-9. 